УДК 517.955.8

\title{
The Properties of the Solutions for Cauchy Problem of Nonlinear Parabolic Equations in Non-Divergent Form with Density
}

\author{
Jakhongir R. Raimbekov* \\ National University of Uzbekistan \\ Yunus Abad-17, 3/66, 10037 \\ Tashkent, Uzbekistan
}

Received 02.04.2014, received in revised form 15.10.2014, accepted 03.04.2014

We investigate the solutions for the following nonlinear degenerate parabolic equation in non-divergent form with density

$$
|x|^{n} \frac{\partial u}{\partial t}=u^{m} \operatorname{div}\left(|\nabla u|^{p-2} \nabla u\right)
$$

We discuss the properties, which are different from those for the equations in divergence form, thus generalizing various known results. Then getting a self-similar solution we show the asymptotic behavior of solutions at $t \rightarrow \infty$. Slow and fast diffusion cases are investigated. Finally, we present the results of some numerical experiments.

Keywords: nonlinear degenerate parabolic equation, non-divergent form, self-similar solution, asymptotic behavior of solutions.

\section{Introduction}

We consider the Cauchy problem for a parabolic nonlinear equation in non-divergent form with density

$$
\begin{gathered}
|x|^{n} \frac{\partial u}{\partial t}=u^{m} \operatorname{div}\left(|\nabla u|^{p-2} \nabla u\right), \quad(x, t) \in \mathbb{R}^{N} \times \mathbb{R}_{+}, \\
\left.u\right|_{t=0}=u_{0}(x) \geqslant 0, \quad x \in \mathbb{R}^{N}
\end{gathered}
$$

for real numbers $p>1,0 \geqslant m<\frac{(p-2)(N+n)+p+n}{p-N}$ and nonnegative integer $n$.

The equation (1) describes many physical problems such as dispersal mechanisms on specials survival, plasma physics, damage mechanics and curve shortening flow, see [11-13].

The equation (1) may be degenerate at the points where $u=0$ and $\nabla u=0$. So we cannot expect to have a classical solution in general, we consider only weak solutions which are nonnegative and in the following weak sense.

Definition 1. A nonnegative function $u(x, t)$ is called a weak solution of (1)-(2) if u satisfies

1) $u \in L^{m+p-1}(\Omega \times(\tau, T)), u_{t} \in L_{l o c}^{2}\left(\Omega_{T}\right),|u|^{m /(p-1)} \nabla u,|u|^{(m-1) / p} \nabla u \in L^{p}\left(\Omega_{T}\right)$ for $\forall \tau \in(0, T)$ and a bounded domain $\Omega \subset \mathbb{R}^{N}$ with smooth boundary, $\Omega_{T}=\Omega \times(0, T)$;

2) $\int_{0}^{T} \int_{\Omega}\left(u \frac{\partial \varphi}{\partial t}-|\nabla u|^{p-2} \nabla u \nabla\left(u^{m} \varphi\right)\right) d x d t+\int_{\Omega} u_{0} \varphi(0, x) d x=0$;

3) $\left.u\right|_{(0, T) \times \partial \Omega} \geqslant 0$;

*raimbekov.jahongir@mail.ru

(c) Siberian Federal University. All rights reserved 


\section{4) for all $0 \leqslant\left.\varphi \in C^{1}\left(\bar{\Omega}_{T}\right) \quad \varphi\right|_{(0, T) \times \partial \Omega}=\left.\varphi\right|_{t=T}=0$.}

The properties of the solution to the problem (1)-(2) depend on the values of the parameters of the equation (1). The cases $m=1$ or $p=2$ (i.e. $p$-Laplacian equation and porous medium equation) were thoroughly studied by many authors (see e.g. $[9,10,18]$ ).

For $0<m<1$, the definition $v(x, t):=(1-m)^{\frac{1-m}{m}} u^{1-m}(x, t)$ transforms $(1)$ into a reactiondiffusion equation with double nonlinearity in divergent form, which has been studied in [1].

The existence of a unique so-called viscosity solution of the Cauchy problem (1)-(2) for $p=2$, was proved in [14]. The authors investigated both viscosity sub-solutions and viscosity sup-solutions for a short time interval. Giving the requirements for the initial values and defining the solutions in a specific way they showed a property of uniqueness for the viscosity solutions which is missing for classic and even other weak solutions.

Indeed, existence of week solutions and also uniqueness have been established in most cases for divergent form equations using various techniques [9,10], but these results do not hold the non-divergent equation (1). Dal Passo and Luckhaus claim that for the case $p=2$ uniqueness fails since for every $T>0$ a weak solution with extinction time $T$. They just got a unique maximal solution and showed that its support remains constant [5]. However, in [12] a counter example was shown by Ughi, who has proved uniqueness of the. This phenomenon was fully discussed later in a cooperative work of these authors [2]. Also, non uniqueness was investigated for $p>1, m=1$ in [17].

The support of solutions of the equation (1) will never expand at $m>1$, while it is known that the equations in divergence form have the property of the finite (or infinite) speed of propagation of disturbance $[5,12,14]$.

The aim of this paper is to find some self-similar solutions, which can be constructed in two ways: forward and backward, then to prove that all solutions satisfying equation (1) has the following asymptotic:

$$
f(\xi) \rightarrow C\left(a-b \xi^{\frac{p+n}{p-1}}\right)^{\frac{p-1}{p+m-2}} \text { at } \xi \rightarrow(a / b)^{(p-1) /(p+n)} \text { if } b>0 \text { and } \xi \rightarrow \infty \text { when } b<0,
$$

where $\mathrm{C}$ is an arbitrary constant.

We will study self-similar solutions of the equation (1) in the form

$$
u(x, t)=(t+1)^{-\alpha} f(\xi),
$$

where $\xi=(t+1)^{-\beta}|x|$ and $\alpha=\frac{1-\beta(p+n)}{m+p-2}$ satisfy the following

$$
f^{m} \xi^{1-N} \frac{d}{d \xi}\left(\xi^{N-1}\left|\frac{d f}{d \xi}\right|^{p-2} \frac{d f}{d \xi}\right)+\beta \xi^{n+1} \frac{d f}{d \xi}+\alpha \xi^{n} f=0 .
$$

In the recent work [7], for the equation (1) without density was obtained the the same selfsimilar equation.

\section{Asymptotic behavior of the self-similar solutions}

In accordance with the statement of the original problem we will consider nontrivial, nonnegative solutions of the equation (4) satisfying the following conditions:

$$
f^{\prime}(0)=0, f(\infty)=0 .
$$

An exact solution for the problem (4)-(5) can be obtained a solution of the following form

$$
f(\xi)=\left(a-b \xi^{\gamma_{1}}\right)_{+}^{\gamma_{2}} .
$$


The solution of this form first was found by Zel'dovich, Kompaneets and Barenblatt in 1950 for porous medium equation [19]. Hence, these type of solutions usually are named as ZKB solutions.

A constant $a$ is arbitrary and positive, but it is advisable to choose it when

$$
a^{\gamma_{2}} \geqslant u_{0}(x), \forall x \in R^{N} \text {. }
$$

Notation $(s)_{+}=\max (0, s)$ is used to show that we are searching the solution with compact support for initial value problem $(4),(5)$.

The constants $b, \gamma_{1}$ and $\gamma_{2}$ will be defined after substituting (6) into (5) which yields

$$
\begin{aligned}
& -\left(b \gamma_{1} \gamma_{2}\right)^{p-1}\left(N-1+\left(\gamma_{1}-1\right)(p-1)\right) \overline{\xi^{\left(\gamma_{1}-1\right)(p-1)-1}\left(a-b \xi^{\gamma_{1}}\right)^{\gamma_{2} m+(p-1)\left(\gamma_{2}-1\right)}}+ \\
& +b^{p} \gamma_{1}^{p} \gamma_{2}^{p-1}(p-1)\left(\gamma_{2}-1\right) \overline{\xi^{\left(\gamma_{1}-1\right)(p-1)-1+\gamma_{1}}\left(a-b \xi^{\gamma_{1}}\right)^{\gamma_{2} m+(p-1)\left(\gamma_{2}-1\right)-1}-} \\
& -\beta b \gamma_{1} \gamma_{2} \mid \xi^{\gamma_{1}+n}\left(a-b \xi^{\gamma_{1}}\right)^{\gamma_{2}-1}+\frac{1-\beta(p+n)}{m+p-2} \overline{\xi^{n}\left(a-b \xi^{\gamma_{1}}\right)^{\gamma_{2}}}=0 .
\end{aligned}
$$

Considering that the first and the third terms are as the second and the fourth ones respectively, we can define $\gamma_{1}$ and $\gamma_{2}$ by equalizing relevant powers,

$$
\left\{\begin{array}{l}
\left(\gamma_{1}-1\right)(p-1)-1=n, \\
\gamma_{2} m+(p-1)\left(\gamma_{2}-1\right)=\gamma_{2}
\end{array}=>\left\{\begin{array}{l}
\gamma_{1}=\frac{p+n}{p-1} \\
\gamma_{2}=\frac{p-1}{p+m-2}
\end{array}\right.\right.
$$

we came to the following form

$$
\begin{aligned}
& {\left[\left(b \frac{p+n}{p+m-2}\right)^{p}(1-m)-\beta b \frac{p+n}{p+m-2}\right] \mid \xi^{\gamma_{1}+n}\left(a-b \xi^{\gamma_{1}}\right)^{\gamma_{2}-1}+} \\
& +\left[\frac{1-\beta(p+n)}{m+p-2}-\left(b \frac{p+n}{p+m-2}\right)^{p-1}(N+n)\right] \sqrt{\xi^{n}\left(a-b \xi^{\gamma_{1}}\right)^{\gamma_{2}}}=0 .
\end{aligned}
$$

This equation is true only if both coefficients are zeros, i.e

$$
\begin{aligned}
& \left\{\begin{array}{l}
\beta=\left(b \frac{p+n}{p+m-2}\right)^{p-1}(1-m), \\
\frac{1-\beta(p+n)}{m+p-2}=\left(b \frac{p+n}{p+m-2}\right)^{p-1}(N+n)
\end{array}=>\right. \\
& =>\left\{\begin{array}{l}
\beta=\frac{1-m}{(N+n)(m+p-2)+(1-m)(p+n)}, \\
b=\frac{p+m-2}{p+n}\left[\frac{1}{(N+n)(m+p-2)+(1-m)(p+n)}\right]^{1 /(p-1)}
\end{array}\right.
\end{aligned}
$$

We defined not only $b$, but also $\beta$ which is positive in case $0<m \leqslant 1$, and negative if

$$
1<m<\frac{(p-2)(N+n)+p+n}{p-N} \text { and } p>N .
$$

Above we considered $p+m-2 \neq 0$. For other case we have to find the solution of this form,

$$
f(\xi)=C e^{\gamma}
$$


By using the above calculations we can obtain the following general exact solution,

$$
\bar{f}(\xi)=\left\{\begin{array}{lc}
\left(a-b \xi^{\frac{p+n}{p-1}}\right)_{+}^{\frac{p-1}{p+m-2}}, & \text { if } m+p-2 \neq 0 \\
\exp (-c \xi)^{\frac{p}{p-1}}, & \text { if } m+p-2=0
\end{array}, \quad \xi=(t+1)^{-\beta}|x|\right.
$$

where $a>0$,

$$
\begin{aligned}
& b= \frac{p+m-2}{p+n}\left(\frac{1}{(p+m-2)(N+n)+(1-m)(p+n)}\right)^{\frac{1}{p-1}}, c=\left(\frac{(p-1)}{p}\right)^{2-\frac{2}{p}}\left[\frac{\beta}{(2)(p-1)}\right]^{\frac{2}{p}}, \\
& \beta= \begin{cases}\frac{1-m}{(p+m-2)(N+n)+(1-m)(p+n)}, & \text { if } m+p-2 \neq 0, \\
-\frac{2}{p}, & \text { if } m+p-2=0,\end{cases} \\
& \alpha= \begin{cases}\frac{1-\beta(p+n)}{m+p-2}, & \text { if } m+p-2 \neq 0, \\
\frac{N}{p(p-1)}, & \text { if } m+p-2=0,\end{cases}
\end{aligned}
$$

are given constants.

We can conclude that the support remains constant in time at $\beta=0$, while for $\beta>0$, the supports keep shrinking and asymptotically go to a single point as $t \rightarrow \infty$ as stated in [7].

\subsection{Slow diffusion (case $\mathbf{m}+\mathbf{p}-\mathbf{2}>0$ )}

The solution (7) is also a sub-solution as it satisfies the condition

$$
f^{m} \xi^{1-N} \frac{d}{d \xi}\left(\xi^{N-1}\left|\frac{d f}{d \xi}\right|^{p-2} \frac{d f}{d \xi}\right)+\beta \xi^{n+1} \frac{d f}{d \xi}+\alpha \xi^{n} f \leqslant 0 .
$$

We will show that the function (7) is an asymptotic of all solutions of the problem (1)-(2) where $p>2-m, 0<m<1$.

We will find solutions to the problem in the following form

$$
f(\xi)=\bar{f}(\xi) \cdot w(\eta), \quad \eta=-\ln \left(a-b \xi^{\frac{p+n}{p-1}}\right),
$$

where $0<w(\eta) \leqslant 1$ for all $\eta$ in $[-\ln (a) ;+\infty)$. It is easy to see that

$$
\xi \rightarrow\left(\frac{a}{b}\right)_{-}^{\frac{p-1}{p+n}} \quad \text { at } \quad \eta \rightarrow+\infty
$$

After the transformation (8) the equation (4) becomes

$$
\begin{aligned}
& w^{m}\left(|L w|^{p-2} L w\right)^{\prime}-\left(\frac{(1-m)(p-1)}{p+m-2}\right) w^{m}|L w|^{p-2} L w+ \\
& +(N+n)\left(\frac{b(p+n)}{(p-1)}\right)^{-1} \frac{b e^{-\eta}}{a-e^{-\eta}} w^{m}|L w|^{p-2} L w+ \\
& +\beta\left(\frac{(p-1)}{b(p+n)}\right)^{p-1} L w+\alpha\left(\frac{(p-1)}{b(p+n)}\right)^{p} \frac{b e^{-\eta}}{a-e^{-\eta}} w=0,
\end{aligned}
$$

where

$$
L w=w^{\prime}-\frac{p-1}{p+m-2} w
$$


At first, we will show that the solution of the equation (4) has finite limit $w_{0}$ at $\eta \rightarrow+\infty$.

Let us take the function

$$
\vartheta(\eta)=|L w|^{p-2} L w .
$$

Then the equation (9) is transformed into

$$
\left\{\begin{aligned}
w^{\prime}= & \frac{p-1}{p+m-2} w+|\vartheta|^{q-2} \vartheta \\
\vartheta^{\prime}= & \left(\frac{(1-m)(p-1)}{p+m-2}-(N+n)\left(\frac{b(p+n)}{(p-1)}\right)^{-1} \frac{b e^{-\eta}}{a-e^{-\eta}}\right) \vartheta- \\
& \quad-\beta\left(\frac{(p-1)}{b(p+n)}\right)^{p-1} w^{-m}|\vartheta|^{q-2} \vartheta-\alpha\left(\frac{(p-1)}{b(p+n)}\right)^{p} \frac{b e^{-\eta}}{a-e^{-\eta}} w^{1-m}
\end{aligned}\right.
$$

Lemma 1. Assume that $0<A_{1} \leqslant A_{2}, \sigma_{1}<\sigma_{2} l \leqslant 0$, and let $\left(w_{1}, \vartheta_{1}\right),\left(w_{2}, \vartheta_{2}\right)$ be the solutions of the system (10) with the initial value conditions $w_{i}\left(\eta_{0}\right)=A_{i}, \vartheta_{i}\left(\eta_{0}\right)=\sigma_{i}(i=1,2)$. If $w_{1}$ and $w_{2}$ are positive in $\left[\eta_{0}, \infty\right)$ then $w_{1}(\eta) \leqslant w_{2}(\eta), \vartheta_{1}(\eta)<\vartheta_{2}(\eta)$ for any $\eta \in\left[\eta_{0}, \infty\right)$.

Proof. As $0<w_{1}\left(\eta_{0}\right) \leqslant w_{2}\left(\eta_{0}\right), \vartheta_{1}\left(\eta_{0}\right)<\vartheta_{2}\left(\eta_{0}\right) \leqslant 0$,

$$
\begin{aligned}
w_{2}^{\prime}\left(\eta_{0}\right)=\frac{p-1}{2-m-p} w_{2}\left(\eta_{0}\right)+\left|\vartheta_{2}\left(\eta_{0}\right)\right|^{p-2} \vartheta_{2}\left(\eta_{0}\right)> & \\
& >\frac{p-1}{2-m-p} w_{1}\left(\eta_{0}\right)+\left|\vartheta_{1}\left(\eta_{0}\right)\right|^{p-2} \vartheta_{1}\left(\eta_{0}\right)=w_{2}^{\prime}\left(\eta_{0},\right) .
\end{aligned}
$$

We came to $w_{2}^{\prime}\left(\eta_{0}\right)>w_{1}^{\prime}\left(\eta_{0}\right)$. Then there must exist a constant $\delta>0$ such that $w_{1}(\eta) \leqslant w_{2}(\eta)$, $\vartheta_{1}(\eta)<\vartheta_{2}(\eta)$ on $\left[\eta_{0} ; \eta_{0}+\delta\right]$. By repeating this process many times we can conclude that $w_{1}(\eta) \leqslant w_{2}(\eta), \vartheta_{1}(\eta)<\vartheta_{2}(\eta)$ conditions true for all $\eta \in\left[\eta_{0} ; \infty\right)$.

Lemma 2. Assume that $0<A_{1} \leqslant A_{2}, 0 \geqslant \sigma_{1} \geqslant \sigma_{2}$, and let $\left(w_{1}, \vartheta_{1}\right),\left(w_{2}, \vartheta_{2}\right)$ be the solutions of the system (10) with the initial value conditions $w_{i}\left(\eta_{0}\right)=A_{i}, \vartheta_{i}\left(\eta_{0}\right)=\sigma_{i}(i=1,2)$. If $w_{1}$ and $w_{2}$ are positive in $\left[\eta_{0}, \infty\right)$ then $w_{1}(\eta) \leqslant w_{2}(\eta), \vartheta_{1}(\eta) \geqslant \vartheta_{2}(\eta)$ for any $\eta \in\left[\eta_{0}, \infty\right)$.

Proof. From the hypotheses we have

$$
\begin{aligned}
& \vartheta_{2}\left(\eta_{0}\right)^{\prime}-\left(\frac{(1-m)(p-1)}{p+m-2}\right) \vartheta_{2}\left(\eta_{0}\right)+(N+n)\left(\frac{b(p+n)}{(p-1)}\right)^{-1} \frac{b e^{-\eta}}{a-e^{-\eta}} \vartheta_{2}\left(\eta_{0}\right)=\vartheta_{2}\left(\eta_{0}\right)^{\prime}= \\
& =-\beta w_{2}^{-m}\left(\frac{(p-1)}{b(p+n)}\right)^{p-1}\left|\vartheta_{2}\right|^{q-2} \vartheta_{2}-\left.\alpha\left(\frac{(p-1)}{b(p+n)}\right)^{p} \frac{b e^{-\eta}}{a-e^{-\eta}} w_{2}^{1-m}\right|_{\eta=\eta_{0}}= \\
& =-\beta\left(\frac{(p-1)}{b(p+n)}\right)^{p-1} w_{2}^{-m}\left(\eta_{0}\right)\left(\left|\vartheta_{2}\right|^{q-2} \vartheta+\frac{p-1}{p+m-2} w_{2}\left(\eta_{0}\right)\right)= \\
& =-\beta\left(\frac{(p-1)}{b(p+n)}\right)^{p-1} w_{2}^{-m}\left(\eta_{0}\right) w_{2}^{\prime}\left(\eta_{0}\right)<-\beta w_{1}^{-m}\left(\frac{(p-1)}{b(p+n)}\right)^{p-1}\left|\vartheta_{1}\right|^{q-2} \vartheta_{1}- \\
& -\left.\alpha\left(\frac{(p-1)}{b(p+n)}\right)^{p} \frac{b e^{-\eta}}{a-e^{-\eta}} w_{1}^{1-m}\right|_{\eta=\eta_{0}} ^{p-1}=-\beta\left(\frac{(p-1)}{b(p+n)}\right)^{-m}\left(\eta_{0}\right) w_{1}^{\prime}\left(\eta_{0}\right)= \\
& =\vartheta_{1}\left(\eta_{0}\right)^{\prime}-\left(\frac{(1-m)(p-1)}{p+m-2}\right) \vartheta_{1}\left(\eta_{0}\right)+(N+n)\left(\frac{b(p+n)}{(p-1)}\right)^{-1} \frac{b e^{-\eta_{0}}}{a-e^{-\eta_{0}}} \vartheta_{1}\left(\eta_{0}\right)=\vartheta_{1}\left(\eta_{0}\right)^{\prime},
\end{aligned}
$$

which means that $\vartheta_{2}^{\prime}\left(\eta_{0}\right)<\vartheta_{1}^{\prime}\left(\eta_{0}\right), w_{2}^{\prime}\left(\eta_{0}\right)>w_{1}^{\prime}\left(\eta_{0}\right)$. Then considering the proof of Lemma 1 we have $w_{1}(\eta) \leqslant w_{2}(\eta), \vartheta_{1}(\eta)>\vartheta_{2}(\eta)$ for all $\eta \in\left[\eta_{0} ; \infty\right)$.

Theorem 1. Let $p>2-m$, then finite solution of the problem (4)-(5) has asymptotic at $\xi \rightarrow\left(\frac{a}{b}\right)_{-}^{\frac{p-1}{p+n}}$ 
Proof. We first show that the solution of the system (10) has finite limit $w_{0}$ at $\eta \rightarrow+\infty$ We know that the function $w_{\eta}$ is bounded. So it is enough to prove that it is monotone nonincreasing in $\left[\eta_{0} ; \infty\right)$. Let us take $w \equiv 1$, we can see that it is a sub-solution from (8). Then for other solutions $w_{1}(\eta)$ we have $w_{1}^{\prime}\left(\eta_{0}\right) \leqslant 0$. That means that any solution is nonincreasing in $\left[\eta_{0} ; \eta_{1}\right)$, for any $\eta_{1}>\eta_{0}$, where difference $\eta_{1}-\eta_{0}$ sufficiently small. By consideration Lemma 1 and Lemma 2 we can find two solutions $w_{1}, w_{2}$ such that $w_{1}\left(\eta_{1}\right)=w_{2}\left(\eta_{0}\right)$. We can conclude

$$
w_{1}\left(\eta_{1}\right) \geqslant w_{1}\left(\eta_{0}\right)
$$

From the arbitrariness of $\eta_{1}$, we see that $w_{1}$ is monotonic in $\left[\eta_{0} ; \infty\right)$. Thus, it has limit at $\eta \rightarrow \infty$. Here taking into consideration that

$$
\lim _{\eta \rightarrow+\infty} \frac{b e^{-\eta}}{a-e^{-\eta}} \rightarrow 0, w^{\prime}=0
$$

in $(9)$ we will get the following algebraic equation at $\xi \rightarrow\left(\frac{a}{b}\right)_{-}^{\frac{2(p-1)}{p}}$,

$$
(1-m)\left(\frac{p-1}{p+m-2}\right)^{p-1} w^{m+p-1}-\beta\left(\frac{(p-1)}{b(p+n)}\right)^{p-1} w=0 .
$$

Calculating the one gives $w=1$, considering (8) the theorem has been proved.

\subsection{Fast diffusion (case $\mathbf{m}+\mathbf{p}-2<0$ )}

In this case we have different families of solutions, oscillating near at $\xi=+\infty$, see for example $[9,10]$. For every of these families there is a constant $\mathrm{C}$ from (3). These kind of solutions are called eigenfunctions [6]. Upper solution for the problem (1)-(2) is obtained by nonlinear splitting [3].

$$
\theta(\xi)=A\left(a+\xi^{\frac{p+n}{p-1}}\right)^{-\frac{p-1}{2-m-p}}
$$

where $A=\left(\frac{p+n}{2-m-p}\right)^{\frac{p-1}{2-m-p}}[(1-m)(p+n)-(N+n)(2-m-p)]^{\frac{1}{2-m-p}}, a>0$.

Theorem 2. Let $p>1+\frac{(n+N)(1-m)}{1-m+n+N}, m<1$, then finite solution of the problem (4)-(5) has asymptotic $f \sim \theta$.

Proof. Let us insert new transformation to (4)

$$
f(\xi)=\theta(\xi) \cdot w(\eta), \quad \eta=\ln \left(a+\xi^{\frac{p+n}{p-1}}\right)
$$

admit that

$$
\eta \rightarrow+\infty \quad \text { at } \quad \xi \rightarrow+\infty
$$

After the transformation (11), we have

$$
\begin{aligned}
& \frac{d}{d \eta}\left(|L w|^{p-2} L w\right)-\left(\frac{(1-m)(p-1)}{2-m-p}\right) w^{m}|L w|^{p-2} L w+ \\
& +(N+n) \frac{p-1}{p+n} \frac{1}{1-a e^{-\eta}} w^{m}|L w|^{p-2} L w+ \\
& +\beta A^{2-p-m}\left(\frac{p-1}{p+n}\right)^{p-1} L w+\alpha A^{2-p-m}\left(\frac{p-1}{p+n}\right)^{p} \frac{1}{1-a e^{-\eta}} w=0
\end{aligned}
$$


where

$$
L w=w^{\prime}-\frac{p-1}{2-m-p} w .
$$

Taking into the consideration that $w(\eta)$ has a finite limit (see the proof of Theorem 1) and

$$
\lim _{\eta \rightarrow+\infty} \frac{1}{1-a e^{-\eta}} \rightarrow 1, w^{\prime}=0
$$

we come to the following algebraic equation

$$
\begin{aligned}
& {\left[(1-m)\left(\frac{p-1}{2-m-p}\right)^{p}-(N+n) \frac{p-1}{p+n}\left(\frac{p-1}{2-m-p}\right)^{p-1}\right] w^{m+p-2}-} \\
& -\beta A^{2-p-m}\left(\frac{p-1}{p+n}\right)^{p-1} \frac{p-1}{2-m-p}+\alpha A^{2-p-m}\left(\frac{p-1}{p+n}\right)^{p}=0
\end{aligned}
$$

at $\eta \rightarrow+\infty$. The last one gives $\mathrm{w}=1$ and on behalf of (11) we have proved that $f \sim \theta$.

\section{Results of numerical analysis and a visualization}

The main difficulty of numerical research for the problem (1)-(2) arises from the nonuniqueness of solutions. To choose right initial data is important on the process of calculations and for testing purposes of numerical analysis we can utilize the exact solution (7) to get initial and boundary values. The asymptotic formula (6) will provide the similarity of the behaviour of solutions to the exact solution (7).

Numerical experiments show properties of solutions clearly. We can see on Fig. 1. extinction solutions for fast and slow diffusion cases. Speeds of perturbation are finite for these cases and we can see how they slow down and maximal speed is at $t=0$.

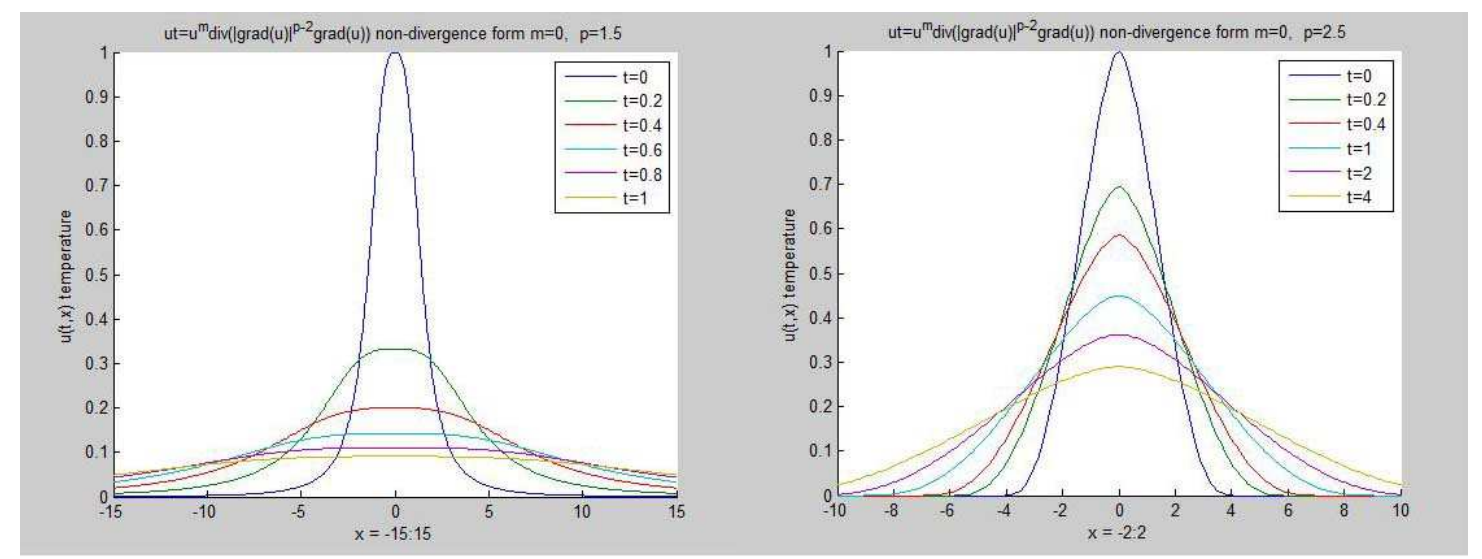

$a$

$\mathrm{b}$

Fig. 1. Divergent cases: a) fast diffusion case; b) slow diffusion case

The figures for non-divergent cases are quite different. Fig. 2 shows that if $\beta=0$ (or $m=1)$, the support of the solution of the problem (1)-(2) remains constant in time. Pay attention to two fixed points on the front of the graph a. If $\beta>0$ (or $m>1$ ), we get shrinking solutions, which asymptotically go to a single point. (Fig. 2, b) It shrinks with a finite speed that also slow down and has maximal speed at $t=0$.

For numerical analysis has been used tridiagonal matrix algorithm. The results of numerical experiments show fast convergence of iteration process (iteration doesn't exceed of two). 


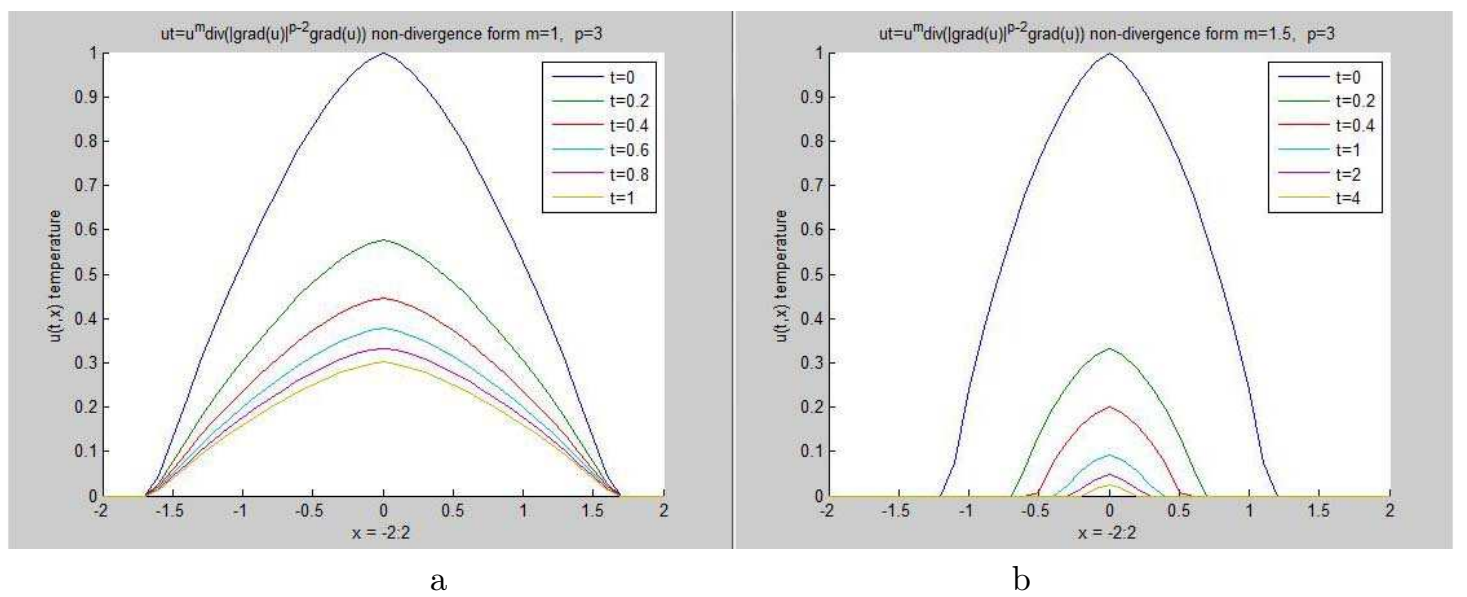

Fig. 2. Non-divergent cases: a) support remains constant; b) support shrinks

\section{References}

[1] M.Aripov, S.A.Sadulaeva, To properties of solutions to reaction diffusion equation with double nonlinearity with distributed parameters, Jour. of Siberian Fed. Univer. Math. \& Phys., 6(2013), no. 2, 157-167.

[2] M.Aripov, Standard Equation's Methods for Solutions to Nonlinear problems, Tashkent, FAN, 1988.

[3] M.Bertsh, R.D.Passo, M.Ughi, Nonuniqueness of solutions of a degenerate parabolic equation, Ann. Mat. Pura Appl. (IV), 161(1992), 57-81.

[4] M.Bertsch, P.Bisegna, Blow-up of solutions of a nonlinear parabolic equation in damage mechanics, Eur. J. Appl. Math., 8(1997), 89-123.

[5] R.D.Passo, S.Luckhaus, A degenerate diffusion problem not in divergence form, Jour. of Diff. Eq., 69(1987), 1-14.

[6] V.A.Galaktionov, S.P.Kurdyumov, A.A.Samarskii, On asymptotic eigenfunctions of the Cauchy problem for a nonlinear parabolic equation, Math. USSR Sbornik, 54(1986), 421-455.

[7] C.Jin, J.Yin, Self-similar solutions for a class of non-divergence form equations, Nonlinear Differ. Equ. Appl. Nodea, 20(2013), no. 3, 873-893.

[8] R.G.Iagar, J.L.Vazquez, Asymptotic analysis for a p-Laplacian evolution equation in an exterior domain, Almeria, San Jose, 2007.

[9] S.Kamin, J.L.Vazquez, Fundamental solutions and asymptotic behaviour for the p-Laplacian equation, Revista Matematica Iberoamericana, 4(1988), no. 2, 339-354.

[10] L.A.Peletier, Z.Junning, Large time behaviour of solutions of the porous media equation with absorption: the fast diffusion case, Nonlinear Analysis, Theory, Methods \& Applications, 17(1991), no. 10, 991-1009.

[11] W.Shu, W.Mingxin, X.C.Hong, A nonlinear degenerate diffusion equation not in divergence form, Z. Angew. Math. Phys., 51(2000), 149-159. 
[12] M.Ughi, A degenerate parabolic equation modeling spread of an epidemic, Ann. Mat. Pura Appl., 143(1986), 385-400.

[13] C.Wang, J.Yin, Shrinking self-similar solutions of a nonlinear diffusion equation with nondivergence form, J. math. Anal. Appl., 289(2004), 387-404.

[14] W.Zhou, Z.Yao, Cauchy problem for a degenerate parabolic equation with non-divergence form, Acta. Mathematica Scienta, 30B(2010), no. 5, 1679-1686.

[15] M.Wiegner, Blow-up for solutions of some degenerate parabolic equations, Differ. Integral Eqs., 7(1994), 1641-1647.

[16] Z.Wenshu, Y.Zheng-an, Behaviors of solutions for a singular diffusion equation, J. Math. Anal. Appl., 327(2007), 611-619

[17] Z.Yao, Z.Wenshu, Nonuniqueness of solutions for a singular diffusion problem, J.Math. Anal. Appl., 325(2007), 183-204.

[18] W.Zhou, Z.Wu, Some results on a class of degenerate parabolic equations not in divergence form, Nonlinear Analysis: Theory, Methods Appl., 60(2005), no. 5, 863-886.

[19] Ya.B.Zel'dovich, A.S.Kompaneets, Towards a theory of heat conduction with thermal conductivity depending on the temperature, In Collection of Papaers Dedicated to 70th Anniversary of A.F. Ioffe, Izd. Akad. Nauk SSSR, Moscow, 1950, 61-72 (in Russian).

\section{Свойства решений одной задачи Коши нелинейного параболического уравнения в недивергентной форме с переменной плотностью}

\section{Жахонгир Р. Раимбеков}

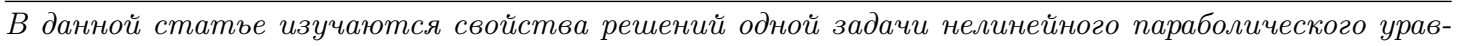
нения в недивергентной борме с переменной плотностъю

$$
|x|^{n} \frac{\partial u}{\partial t}=u^{m} \operatorname{div}\left(|\nabla u|^{p-2} \nabla u\right) .
$$

Обсуждаются свойства решенийб отличных от тех, которые применимы для уравнений в дивергентной форме. Основная цель данной статьи - анализ асимптотических поведений на основе полученных автомодельных решений для случая быстрой и медленной диффузии. Численный анализ решений был получен на основе тех же автомоделъных приближений, визуализачия которых отражает ряд свойствб которые обсуждаются в статъе.

Ключевые слова: нелинейное вырождающееся параболическое уравнение, недивергентная форма, автомодельное решение, асимптотика решений. 